\section{S3A:6 MICROPARTICLES WITH MITOCHONDRIAL MOLECULES AND IMMUNOGLOBULINS ASSOCIATE WITH ACTIVE DISEASE IN SYSTEMIC LUPUS ERYTHEMATOSUS}

${ }^{1} \mathrm{~F}$ Mobarrez, ${ }^{1} \mathrm{E}$ Fuzzi, ${ }^{1} \mathrm{I}$ Gunnarsson, ${ }^{2} \mathrm{D}$ Pisetsky, ${ }^{1} \mathrm{E}$ Svenungsson. ${ }^{1}$ Unit of Rheumatology, Department of Medicine, Solna, Karolinska Institutet, Karolinska University Hospital, Stockholm, Sweden; ${ }^{2}$ Department of Medicine, Duke University Medical Centre, North Carolina, Durham, USA

\subsection{6/lupus-2018-abstract.13}

Background/purpose Systemic lupus erythematosus (SLE) is a prototypic autoimmune disease characterised by immune complexes, especially those with nuclear molecules bound by antinuclear antibodies. Although the source of these antigens is not fully known, increased apoptosis and defective clearance of dead cells have been proposed. During apoptosis and cell activation, microparticles (MPs) are released extracellularly. MPs are small membrane-bound particles that can contain molecules arising from both the nucleus and cytoplasm. Moreover, these MPs are often covered with immunoglobulins, thus forming MP-immune complexes (mpICs).

Methods Plasma samples from 47 patients with SLE and 24 healthy controls were investigated. MPs and mpICs were analysed by flow cytometry and defined by size; MPs $(<0.7 \mu \mathrm{m})$ or mpICs $(>0.7 \mu \mathrm{m})$. Samples were labelled with MitoTracker deep red FM to investigate mitochondrial content. Flow cytometry was also used to assess outer mitochondria markers Tom-20 and hexokinase I, as well as the presence of IgG.

Results Levels of both MPs and mpICs in SLE patients were significantly elevated compared to controls (figure 1A). Although MP concentrations were higher than those of mpICs, mpICs contained more mitochondria compared to MPs (figure 1B). mpICs also displayed $\operatorname{IgG}$ and exposed the outer mitochondria markers. The number of mpICs containing mitochondria correlated strongly to the presence of antidsDNA antibodies (figure 2) as well as to levels of TNF $\alpha\left(\mathrm{r}^{2}\right.$ $0.2, \mathrm{p}<0.05)$, IL-6 $\left(\mathrm{r}^{2} 0.23, \mathrm{p}<0.01\right)$ and IL-7 $\left(\mathrm{r}^{2} 0.24\right.$, $\mathrm{p}<0.01)$. Moreover, patients with active renal disease had significantly higher levels of IgG-coated mpICs containing mitochondria. Patients with SLAM above 6, had significantly higher numbers of mpICs containing mitochondria and exposing outermitochondria markers Tom-20 and hexokinase I.

Conclusion MPs and mpICs are significantly more abundant in the blood of SLE patients compared to controls. Importantly, the majority of the mitochondria were present in the larger mpIC sup-population. Moreover, mpICs that contain mitochondria also display outer mitochondria markers, with levels

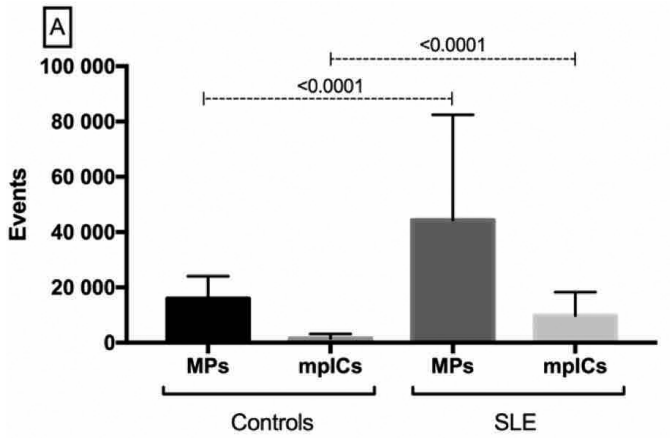

correlating with levels of anti-dsDNA antibodies, disease activity and pro-inflammatory cytokines. mpICs may therefore contribute to SLE pathogenesis, with mitochondria representing a source of cell antigens that can trigger innate and adaptive immune responses as well as deposit in the tissue.

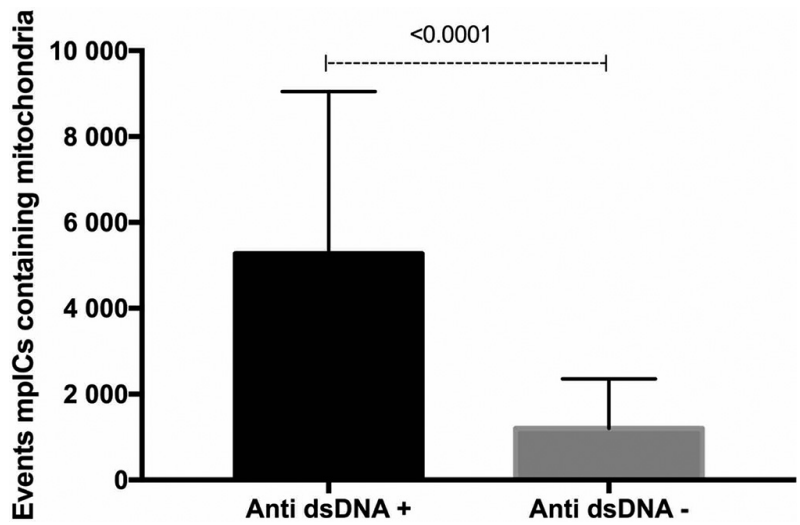

Abstract S3A:6 Figure 2 SLE patients with anti-dsDNA antibodies have higher number of $\mathrm{mplCs}$ contacting mitochondria

\section{S3A:7 NEUTROPHIL EXTRACELLULAR TRAPS MARKERS ARE ELEVATED IN PATIENTS WITH SYSTEMIC LUPUS ERYTHEMATOSUS}

1I Jeremic, ${ }^{2} \mathrm{O}$ Djuric, ${ }^{3} \mathrm{~B}$ Bonaci-Nikolic. IInstitute of Rheumatology, Belgrade, Serbia; ${ }^{2}$ Institute of Epidemiology, Belgrade, Serbia; ${ }^{3}$ Institute of Allergy and Clinical Immunology, Belgrade, Serbia

\subsection{6/lupus-2018-abstract.14}

Introduction Impaired removal of apoptotic waste in patients with systemic lupus erythematosus-SLE has been long known as important factor that trigger autoimmune response. Neutrophil extracellular traps could be another source of autoantigens in patients with SLE.

Methods We analysed sera from 84 SEL patients (60 patients had one sample and 24 patients were followed 2 or 3 times) and 50 healthy blood donors. Serum levels of myeloperoxidase, B-cell activating factor-BAFF, cell free DNA, complement components C3 and C3, antibody to dsDNA by CLIFT end ELISA assays, netolitic activity and DNAse I were measured.

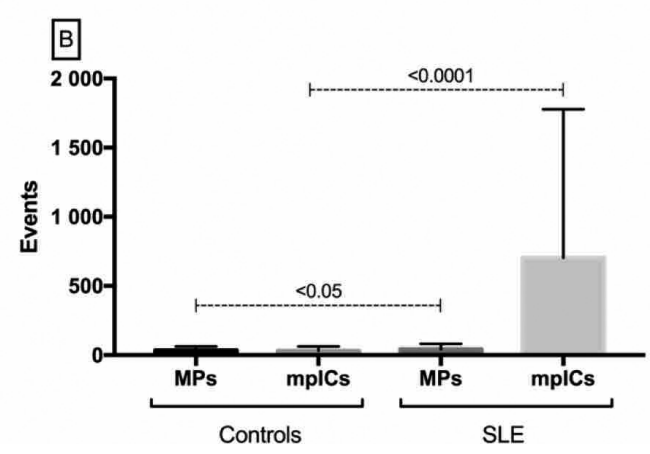

Abstract S3A:6 Figure 1 Microparticles (MPS) and MPS in immune complexes (mplCs) in patients with systemic lupus erythematosus and healthy controls. (A) Number of total MPs and mplCs defined by size and complexity by flow cytometry, in healthy controls and SLE. (B) Number of total MPs and mplCs containing mitochondna (labelled with MitoTracker) $n$ healthy controls and SLE 